\title{
Diseases and health risks associated with obesity
}

\author{
Walter Milano ${ }^{1}$ and Anna Capasso ${ }^{2 *}$ \\ ${ }^{1}$ Mental Health Unit District 24 ASL Napoli 1 Center, Italy \\ ${ }^{2}$ Department of Pharmacy, University of Salerno, 84084-Fisciano, Italy
}

\section{Epidemiology}

Obesity is an evolutive, chronic and recurring medical condition that consists of a pathological accumulation of adipose tissue, in absolute values and percentages, in relation to the lean mass, to such an extent as to negatively affect the state of health. It is a real metabolic disease, which compromises the regulation of appetite and energy metabolism. Obesity is the leading cause of preventable death worldwide, with increasing prevalence in adults and children, and is considered to be one of the most serious public health problems of the $21^{\text {st }}$ century. According to estimates published recently [1] more than 1.5 billion people are overweight or obese and of these 200 million men and 300 million women are obese. So much so that the World Health Organization (WHO) introduced in 2001 the expression globesity, crasis between the English words of obesity and globality, to indicate the global epidemic of obesity on a planetary level. In 2010 more than 5 million children under the age of 5 were already overweight or obese and over $60 \%$ of the world's population lives in countries where overweight and obesity kill more by now than malnutrition [1]. In fact, it is noted that in the richer countries, for the first time, average life expectancy begins to decline, perhaps above all due to the effect of obesity. Currently, in half of the 34 member countries of the OECD (Organization for Economic Co-operation and Development) the total number of overweight and obese people exceeds the number of normalweighted or underweight people. In fact, therefore, in many countries the concept of normopeso does not correspond more to normality in a purely statistical sense [2]. The highest statistical tips are found in the Federated States of Micronesia and in one of them, Kosrae, on a population of just over 8000 inhabitants, $88 \%$ of adults are overweight, $59 \%$ are obese and $24 \%$ are severely obese (BMI> 35). In Louisiana and Mississippi, US states, one in three people is obese, respectively 33.4 and $34.9 \%)$. Regarding the WHO European Region, in 2008, over 50\% of the adult population were overweight and about $23 \%$ of women and $20 \%$ of men were obese [3].

\section{Clinical Events and Comorbility}

Overweight and obesity are the cause of physical disability, reduced working capacity and predispose the onset of numerous chronic diseases. Obesity increases the risk of many physical and mental illnesses. It is mainly associated with metabolic syndromes, combinations of medical disorders that include type 2 diabetes mellitus, hypertension, hypercholesterolemia and hypertriglyceridaemia. In general, the health consequences of obesity fall into two broad categories: the diseases attributable to the effects of increased body fat (such as osteoarthritis, obstructive sleep apnea, social stigmatization) and those due to the increase in the number of fat cells (diabetes, dyslipidemia, cancer, cardiovascular disease, non-alcoholic fatty liver disease) [4]. The correlation between obesity and specific conditions varies; one of the highest concerns type 2 diabetes: the excess of adiposity is in fact at the base of $64 \%$ of cases of diabetes in men and $77 \%$ of cases in women [5]. The increase in fat mass alters the response to insulin, favoring insulin resistance; the increase in adipose tissue also generates a proinflammatory state $[6,7]$ and a prothrombotic $[8,9]$. Finally it should be noted that the presence of obesity increases the risk of development of some cancers such as endometrial cancer, colon-rectal cancer, gallbladder and breast [10]. The risk of developing diseases increases with increasing body mass index.

\section{Quality of Life of Obese Patients}

Obesity is frequently associated with a worse quality of life. The diseases associated with obesity mentioned above can contribute to reducing the quality of life since they reduce the physical abilities of patients (let's think for example of cardiological, respiratory or osteoarticular problems) and involve chronic pharmacological therapies that can in turn be associated with side effects. Some disorders such as depression, infertility or impotence can also further reduce the quality of life of patients in relation to interference with interpersonal relationships. Finally, within social life there is a wide range of repercussions such as discrimination in the workplace, difficulties in everyday situations such as the search for clothing, the way to travel to daily activities such as washing or climbing stairs.

\section{Mortality Data}

If we consider the diseases and health risks associated with obesity, it is clear that obesity can be an important factor in mortality. It is believed that every year in Europe 320,000 people die from causes related directly to obesity; the mortality correlated to excess weight therefore represents a serious public health problem where about $7.7 \%$ of all causes of death are due to excess weight. Life expectancy in the severely obese population is reduced, with a shortening of life expectancy of about 7-10 years, with a risk of death that increases with the increase in body mass index and abdominal circumference [11]. Assuming that the association between high body mass index and mortality is largely causal, the proportion of premature deaths that could be avoided by changing the lifestyle and reducing weight would be about one in seven in Europe and one in 5 in North America. Furthermore, the excess risk for premature death, ie before 70 years

Correspondence to: Anna Capasso, Department of Pharmacy, University of Salerno, 84084-Fisciano, Italy, E-mail: annacap@unisa.it

Key words: Diagnosis, epidemiology, obesity, quality of life, surgical interventions, treatment

Received: February 15, 2018; Accepted: March 05, 2018; Published: March 09, 2018 
of age, among those who are overweight or obese is about three times greater in men than in women. This is consistent with the fact that obese men have higher insulin resistance and a higher risk of diabetes than women [12].

\section{Diagnosis}

Obviously the discrepancy between excess and energy expenditure remains the basis for attempting to explain the onset of excess weight but can not fully explain the mechanisms underlying weight gain. That obesity has a hereditary component is a known fact, but it is only a few decades ago that genetic research has begun to define the role of biological inheritance with respect to environmental influences in the regulation of body weight. For the common obesity the genetic influences seem to express themselves through susceptibility genes that significantly increase the risk of developing a condition of marked overweight but they are not all indispensable for its expression and alone are certainly not sufficient to determine it. Apart from the rare monogenic obesity and obesity related to other genetic syndromes, such as Prader-Willi syndrome or Albright or Bardet-Biedl syndrome, the genes involved are certainly very numerous. From studies on human obesity and especially through animal models, hundreds of candidate genes have been identified, but at present it is not yet clear how genetic variables can interact with individual responses to nutrition and disorders related to different feeding modalities. Obesity, like most chronic degenerative diseases, recognizes a multifactorial genesis, where inheritance and the environment are associated in an intricate manner, albeit in ways that are still partially unknown. Obesity appears at the moment as a genetically complex condition that tends to be associated with a multitude of complications, such as insulin resistance, type 2 diabetes, dyslipidemia, arterial hypertension, etc. These, in turn, are certainly influenced by lifestyle and eating habits but are also linked to genetic predispositions, often independent of one another and very difficult to disentangle and identify [13]. It would therefore be advisable to use the term in the plural, obesity; a term that better understands the different phenotypic expressions collected, at the moment, in the generic definition of obesity.

The measurement of the distribution of body fat can be performed by different methods: from the measurement of skin folds, to the measurement of the waist circumference or its relationship with the circumference of the hips, or with more sophisticated techniques such as ultrasound, axial tomography or magnetic resonance. More simply, but still in a statistically effective manner, the classification of the population based on weight is made using the body mass index (BMI = body mass index, according to the Anglo-Saxon expression), which is considered the most representative of the presence excess body fat. The BMI is calculated according to the following formula: $\mathrm{BMI}=$ weight (in $\mathrm{kg}$ ) / square of height (in meters). The weight classes for adults indicated by the BMI are: $<18.5$ underweight; $18.5-24.9$ normal weight; 25-29.9 overweight; $>30$ obesity. Naturally there are differences related to sex; with the same BMI, women tend to have more body fat than men, as well as older people compared to young people. Moreover, those who have a physical body can weigh more precisely thanks to the highly developed muscle mass, but do not fall for this in the overweight or obesity category. For people under 19 years, the growth curves of the WHO and the threshold values recommended by the International Obesity Task Force (IOTF) that take into account age and gender are used for weight classification.

\section{Prevention and Screening}

Living nowadays, in an obeseogenic environment, the treatment of obesity becomes complex and easily recurrent. It is therefore necessary to invest in prevention tools that reduce the impact of the environment on individuals. Awareness-raising campaigns on the general population, above all aimed at young people to adopt healthy lifestyles, thanks to a correct diet and adequate physical activity, can allow you to control your weight and avoid exceeding the levels of risk. Selective psychoeducational intervention programs aimed at groups of people presenting risk factors significantly higher than average and above all interventions aimed at individuals at high risk, carriers of biological markers or premorbid symptoms appear even more useful. Particularly effective are programs dedicated to subgroups of children or adolescents at greater risk to which primary and secondary prevention interventions of a selective type and indicated to their age group are applied, which allow to save resources and increase effectiveness with respect to interventions. of a universalistic type. Useful and reachable objectives through primary prevention could be characterized by ensuring easy access to consultation and care services, counseling to families of subjects at risk, dissemination of basic knowledge on health promotion and protection, improving the communication flow between the institutions responsible for prevention (schools, local health agencies, general practitioners, pediatricians of free choice, press and information agencies, voluntary and consumer associations, etc.) and users [13].

\section{Treatment}

The high clinical complexity and the comorbidities connected to obesity necessitate an approach, as for other eating disorders, multidimensional and interdisciplinary that involves a wide range of skills: nutritional, internal, psychiatric and surgical and therefore of different professional figures, Last but not least the general practitioners who know better the patients and the family and social environments in which they live and interact, all involved in interconnected teams and oriented to a punctual management of clinical outcomes. The task of obesity therapy is not only linked to weight loss but also to the prevention or treatment of related diseases. Naturally, weight loss is a substantial condition but must be assessed not only on the extent of weight loss but also on clinical and psychological parameters and on the quality of life of the obese patient. Weight reduction, although modest, is a useful tool and has more value the longer it remains stable over time. The treatment of obesity is based on a stable change over time of the alimentary behaviors and of the psychic processes connected to them. To date, nutritional intervention remains the foundation in the treatment of obesity; the reduction of the caloric intake allows, at least in the short term, to reduce the adipose mass but already relatively quickly induces a reduction of the metabolism, through energy-metabolic regulation mechanisms that tend to energy saving [14]. These defense mechanisms support, first a reduction of the decline, and then a substantial return to weight before the diet, the socalled weight cycling syndrome, which involves frustration, a sense of inability and devaluation. Therefore, it is essential to also promote an increase in energy expenditure, through a balanced and progressive increase in physical activity, compatible with the patient's clinical condition, so as to keep active the metabolic, cardio-respiratory and even psychic processes. In an obese patient already walking for 30-45 minutes a day can allow, together with a balanced diet, an acceptable weight loss around $10 \%$ of the initial weight in a few months. Since obesity is a chronic condition for these results to be maintained over time, it should be accompanied by support programs, such as integrated psychoeducational or psychotherapeutic interventions of a cognitive or problem-solving type, aimed at modifying the lifestyle in certain types of patients. 
Also the use of drugs, which can facilitate the reduction of caloric intake or energy expenditure, could be useful in obese patients with BMI above 30, even if at present there is still no ideal drug. Beyond all the various fanciful and sometimes dangerous combinations of drugs, it is possible to use some molecules, recognized as valid by the national regulatory body, to be combined with diet and exercise, which can help weight loss. At the moment only two molecules are available in Italy: the orlistat, which inhibits gastrointestinal lipases, reduces the absorption of fats and the liraglutide that is part of a new generation of analogous GLP-1 drugs, able to regulate the metabolism of the glucose and mimics the way the intestine communicates signals to the brain to regulate appetite, reducing food intake. Another opportunity in the treatment of large obesity, BMI $>40$ or with $\mathrm{BMI}>35$ in the presence of associated co-morbidities, is offered by bariatric surgery that allows to determine a significant weight loss in the long term. At least two-thirds of the obese pathological subjects who have chosen bariatric surgery can lose about $50 \%$ of the excess weight over 10 years and beyond when they are motivated to do so and adhere to the therapy. Bariatric surgery has a particularly advantageous cost / benefit ratio (from the first year of treatment), and often allows a significant saving on socio-health costs compared to the conservative approach [15].

\section{Surgical Interventions}

\section{Interventions that limit the introduction of food}

a. mainly mechanical action (restrictive interventions): adjustable gastric banding, vertical gastroplasty and sleeve gastrectomy.

b. Primarily functional action: gastric bypass and variants

Interventions that limit energy absorption: biliopancreatic diversion and duodenal switch [16].

The best results are obtained if the pre-operative and post-operative intervention of the educational and psychological paths, individual or group, that support the patient for a sufficiently long period [17] is associated.

A further therapeutic approach is the multidisciplinary rehabilitation of obesity, which is often reserved for high-grade obesity or with significant complications; this system is based on management by a group of more professionals (internists, nutritionists, psychiatrists, psychologists, dietitians, physiotherapists and nurses, etc.) in the context of multiple settings (outpatient clinic, residential rehabilitation, etc.). This approach tries to reconstruct correct eating habits in the patient, improve his ability to manage body weight, reactivate muscle structures and recover joint mobility, improve the cardiovascular and respiratory system, increase energy expenditure, increase the ratio of lean mass / fat mass and reduce body weight.

\section{Assistance Network}

Obesity must be considered a real disease that must be treated to live longer and with a better quality of life. Therefore, a significant and important cultural and clinical transition is necessary. It is therefore necessary to formalize and implement at national level a more advanced, correct and adequate criterion for the problems of overweight or obese people, structuring a specific Diagnostic Therapeutic Assistance Path (DTAP), as for other eating disorders, organized as a network multidisciplinary with professionalism ranging from the clinical psychologist to the bariatric surgeon, from the pediatrician to the nurse, from the general practitioner to the internist, all involved in teams integrated with regional hubs and various spokes at the local level in the various ASLs. In this way, all patients would be guaranteed the transition from "care episodes" to an approach based on "pathways of care and assistance". This DTAP model would favor not only a multidisciplinary care of the patient but also a whole series of virtuous processes, in a suitable environment, universalism in access, sustainability and economic rationality, risk reduction and finally continuity of care. It could also be very useful to involve the associations of patients and their family members who would also guarantee the voice and needs of the users, to avoid that many people turn to unqualified facilities or operators or even to the many selfstyled healers and sellers of false hopes [18-20].

\section{Conclusions}

The concept of obesity has an ancient history: the expression obesity (obesitas) was used for the first time, almost 2000 years ago, by Aulo Cornelio Censo to indicate an excess of fat but the excess fat had already been described in Egyptian papyri around $1500 \mathrm{BC}$ and from Hippocrates in the 4th century BC Naturally, they were problems linked to small sections of the population, the nobles and the rich, who had access to great food availability and did not need to work; something uncommon in times of war, famine and pestilence. The substantial point is related to the dyscrasia that has developed in recent decades in which increasingly complex societies have been built, in which access to food has become easier while the mechanization of work and transport has led to an ever-lower expenditure of power. Basically, the genetic / biological fund of man struggles not a little to adapt to modernity. Structured to save energy and survive the phases of nutritional deficiency, the human metabolic machine must row countercurrent in an era in which the energy excess compared to consumption is practically the norm. The evolution of millennia has been displaced by the new standards of life. But in spite of technological and cultural advances in eating, modern phylogenetic behavioral residues are still resisting in modern humans, such as eating compulsively large quantities of food in the shortest possible time. It is a primordial mechanism, developed over time, to allow the body to take energy, when available, in greater quantities than necessary to balance the balance of the moment, signaled by the feeling of satiety. The goal was to allow to store reserves for moments of energy shortage. Operation once providential but currently not very suitable, rather unsuitable compared to the excessive energy availability available today for a large slice of the world population. Ancestral behavior is present in binge eating, mainly related to states of emotional stress and psychological disorders. The same low-calorie diets, so current nowadays, represent for the metabolic balance of the organism, a condition of distress such as to favor, in the medium-long period, an alteration of the control on natural hunger / satiety devices, until at the appearance of compulsive feeding phenomena (binge eating). Dieting, or dieting on a diet, is a major cause of eating disorders, particularly Binge Eating Disorder and obesity. Hence the well-known paradox: diets eventually make you fat. The main treatment against obesity consists in the association of diet and exercise. An accurate diet can cause weight loss in the short term; however maintenance is often difficult and requires constant effort in physical exercise as well as proper nutrition. The success rate of longterm weight maintenance with lifestyle change ranges from 2 to $20 \%$. As for drugs, scientific research has always been working to identify new molecules to be associated with the diet. At the moment the doctor has numerous drugs to treat diseases associated with obesity but with regard to the treatment of obesity he has at his disposal only drugs able to act on the reduction of fat absorption that can find indications in selected cases. On the market there are also various herbal products, 
supplements or cocktails of various products that patients can easily find but which are often ineffective if not even in some cases highly detrimental to health.

\section{References}

1. WHO (2015) Obesity and Overweight: fact sheet.

2. OECD (2012) Obesity Update.

3. WHO (2013) Country profiles on nutrition, physical activity and obesity in the 53 WHO European Region Member States.

4. Haslam DW, James WP (2005) Obesity. Lancet 366: 1197-1209. [Crossref]

5. Kopelman PG, Caterson ID, Stock MJ, Dietz WH (2005) Clinical obesity in adults and children. Blackwell Publishing, pp: 3-11.

6. Shoelson SE, Herrero L, Naaz A (2007) Obesity, inflammation, and insulin resistance. Gastroenterology 132: 2169-2180. [Crossref]

7. Shoelson SE, Lee J, Goldfine AB (2006) Inflammation and insulin resistance. J Clin Invest 116: 1793-1801. [Crossref]

8. Bray GA (2004) Medical consequences of obesity. J Clin Endocrinol Metab 89: 25832589. [Crossref]

9. Dentali F, Squizzato A, Ageno W (2009) The metabolic syndrome as a risk factor for venous and arterial thrombosis. Semin Thromb Hemost 35: 451-457. [Crossref]
10. Calle EE, Rodriguez C, Walker-Thurmond K, Thun MJ (2003) Overweight, obesity, and mortality from cancer in a prospectively studied cohort of U.S. adults. $N$ Engl $J$ Med 348: 17. [Crossref]

11. WHO (2013) Country profiles on nutrition, physical activity and obesity in the 53 WHO European Region Member States.

12. The Global BMI Mortality Collaboration (2016) Body Mass Index and all-cause mortality: individual partecipant data meta-analysis of 239 prospective studies in four continents. Lancet 388: 776-786. [Crossref]

13. Bosello, Cuzzolaro "Obesità" il Mulino 2013

14. Bosello, Di Francesco "L' alimentazione" il Mulino 2007

15. Capizzi D, Capizzi FD (2011) “Obesità e chirurgia” CG Edizioni Medico Scientifiche.

16. SIO e ADI "Standard italiani per la cura dell'obesità" 2012.

17. Beck NN, Johannsen M, Støving RK, Mehlsen M, Zachariae R (2012) Do postoperative psychotherapeutic interventions and support groups influence weight loss following bariatric surgery? A systematic review and meta-analysis of randomized and nonrandomized trials. Obes Surg 22: 1790-1797. [Crossref]

18. Milano W (2009) L'obesità. Manuale di sopravvivvenza. Giovanni Fioriti Ed.

19. Molinaro E, Riva G (2004) Psicologia clinica dell'obesità. Bollati Boringhieri.

20. Arnold M, Leitzmann M, Freisling H, Bray F, Romieu I, et al. (2016) Obesity and cancer: An update of the global impact. Cancer Epidemiol 41: 8-15. [Crossref]

Copyright: $@$ 02018 Milano W. This is an open-access article distributed under the terms of the Creative Commons Attribution License, which permits unrestricted use, distribution, and reproduction in any medium, provided the original author and source are credited. 